\title{
Les Suds rêvés de Julien Green, sous la direction d'André-Alain MORELLO
}

\section{Paola Codazzi}

\section{OpenEdition}

\section{Journals}

\section{Edizione digitale}

URL: http://journals.openedition.org/studifrancesi/15831

DOI: 10.4000/studifrancesi.15831

ISSN: 2421-5856

\section{Editore}

Rosenberg \& Sellier

\section{Edizione cartacea}

Data di pubblicazione: 1 décembre 2018

Paginazione: 529-530

ISSN: 0039-2944

\section{Notizia bibliografica digitale}

Paola Codazzi, «Les Suds rêvés de Julien Green, sous la direction d'André-Alain morelto», Studi Francesi [Online], 186 (LXII | III) | 2018, online dal 01 janvier 2019, consultato il 06 janvier 2021. URL: http:// journals.openedition.org/studifrancesi/15831 ; DOI: https://doi.org/10.4000/studifrancesi. 15831

Questo documento è stato generato automaticamente il 6 janvier 2021.

\section{(a) $\odot \Theta$}

Studi Francesi è distribuita con Licenza Creative Commons Attribuzione - Non commerciale - Non opere derivate 4.0 Internazionale. 


\title{
Les Suds rêvés de Julien Green, sous la direction d'André-Alain MORELLO
}

\author{
Paola Codazzi
}

\section{NOTIZIA}

Les Suds rêvés de Julien Green, sous la direction d'André-Alain MORELLO, «Littératures», 76, 2017, $203 \mathrm{pp}$.

1 Pubblicato nel giugno 2017, il dossier di questo numero di «Littératures», curato da André-Alain Morello, è dedicato a Julien Green e al tema del "Sud", declinato in due filoni differenti: il primo è il "Sud" americano, luogo delle origini; il secondo è quello europeo, composto da quei Paesi che sono stati destinazione di viaggio e oggetto di fascinazione (l'Italia, la Spagna e la Grecia). Il volume comprende inoltre una sezione «Varia» e una sezione di «Comptes rendus» di opere critiche recenti.

I primi tre articoli ci conducono a esplorare Savannah, in Georgia, città natale della madre di Green, e Charlottesville, in Virginia, dove il diciannovenne scrittore frequenta per tre anni l'Università. È per motivi di studio che il giovane Julian/Julien si reca per la prima volta negli Stati Uniti ed è in quest'occasione che il "Sud" immaginato incontra il "Sud" reale, diventando materia letteraria. L'articolo di Emmeline GROS (pp. 17-27) invita il lettore a riflettere sul modo in cui queste due immagini si sovrappongano nell'opera narrativa e teatrale dell'autore, dove i paesaggi americani sono qualcosa di più di un semplice sfondo. Michel DYÉ conduce la propria riflessione in questa stessa direzione (pp. 28-41), comparando il "Sud" di Green a quello di François Mauriac, scrittore che ha trovato nel territorio di Bordeaux, luogo di provenienza della sua famiglia, la sua più grande fonte di ispirazione. Anche Enrico GUERINI (pp. 43-53) insiste sull'importanza dello spazio americano nell'imaginario di Green, ma in una prospettiva diversa: attraverso l'analisi dell'opera Sud, l'A. cerca di spiegare come la scelta di un luogo lontano, a livello geografico e storico - la vicenda si svolge durante la Guerra di Secessione -, sia legata alla volontà dello scrittore di interporre una certa distanza tra 
la scène e il pubblico, per poter parlare più liberamente di alcuni temi (tra cui quello dell'omosessualità).

Nella seconda parte del volume, il "Sud" prende forme molto diverse, al punto da dare origine a una pluralità di rappresentazioni e immagini. L'articolo di Daniela FABIANI (pp. 57-66) tratta di un testo piuttosto breve, Florence avec Julien Green. Sebbene queste pagine siano pensate come un récit de voyage, il loro carattere prettamente letterario risulta evidente, al punto da spingere l'A. a considerare lo scritto come un'autobiografia. Che si tratti di Firenze o di Roma, le conclusioni sono molto simili. Frédéric CANOVAS (pp. 67-77) riprende dettagliatamente l'insieme di considerazioni che Green dedica alla capitale d'Italia, mostrando le reazioni contradditorie del viaggiatore di fronte ai suoi monumenti, specchio delle sue inquietudini personali. Altre città della penisola lasciano nello scrittore un ricordo indelebile: come ricorda Annie BRUDO (pp. 79-92), Green osserva entusiasta il Duomo di Milano, la Cattedrale di Orvieto e commenta dettagliatamente i magnifici mosaici di Sant'Apollinare a Ravenna, alla ricerca delle origini della propria fede. È questa stessa ricerca che orienta lo sguardo dell'autore sulla Spagna, destinazione di viaggio in gioventù (1919) e in età matura (1978-79). Come sottolinea Dominique BONNET (pp. 93-108), questo paese profondamente ambivalente non ha mai completamente sedotto Green, a meno che non si consideri la "géographie singulière» (p. 108) che si trova rappresentata nei dipinti di Salvador Dalì: questa Spagna onirica, immaginaria e immaginata, è quella che più lo ha affascinato. Lo stesso discorso, come osserva André-Alain MORELLo (pp. 109-119), sembra valere anche per la Grecia. Lo spazio geografico "reale" non ha quasi nessun rapporto con la Grecia di Green, che è una Grecia ideale, un "Sud" che appare intrinsecamente legato al "Sud" americano, e più particolarmente all'Università della Virginia, tempio di cultura classica.

4 La terza e ultima parte del dossier consacrato a Julien Green è composta da un articolo di Louis BALADIER (pp. 123-136) che, attraverso l'analisi dell'opera letteraria, mostra la tensione esistente tra ici e ailleurs, tra sensualità e misticismo. Più che sul "Sud", si tratta qui di interrogarsi, come indicato dal titolo, su altri spazi - «autres espaces»che i personaggi aspirano a raggiungere per realizzare i propri desideri, i propri sogni.

La sezione "Varia», per quanto lontana dal tema del dossier, rimane in qualche modo legata a esso attraverso lo sviluppo di una riflessione sui concetti di "frontiera" e di “confine". Théo soula (pp. 139-159) e Hannes DE VRIESE (pp. 161-171) si interrogano da punti di vista diversi sullo spazio urbano: il primo riflette sulla separazione tra centro e periferia analizzando la rappresentazione della città di Parigi nei testi di Jacques Réda; il secondo studia la geografia del Cycle de Marie di Jean-Philippe Toussaint, dove l'isola d'Elba, protetta dal mare, rappresenta l'unico luogo in cui la bellezza possa rifugiarsi. Il contributo di Andrei MINZETANU (pp. 174-195) si inserisce anch'esso all'interno di un discorso sulla "frontiera", ma senza alcun riferimento allo spazio geografico: l'articolo tratta infatti della trasformazione che l'opera letteraria subisce quando il lettore se ne appropria, attraverso un processo di scomposizione del testo in citazioni (spesso errate). Le parole, e le idee, viaggiano al punto che anche il più pigro, come scrive Green, «finit par en absorber quelque chose». 